\title{
Nociceptive Reflex in Patients With Schizophrenia: A Case-Control Study
}

\author{
Hosna Elshony a, b, Lamyaa G. Al Hamrawy ${ }^{\mathrm{a}}$
}

\begin{abstract}
Background: Diminished pain sensitivity has been reported in patients in the acute phase of psychosis, as well as in stable patients. The cause of hypoalgesia in those patients is unknown. The aim of this study was to investigate the sensory thresholds, pain modalities, or other factors contributing to the perception or expression of physical pain in patients with schizophrenia (SCZ).
\end{abstract}

Methods: This case-control study was conducted on 24 patients with SCZ and 12 healthy controls. Patients with SCZ were assessed by the Structured Clinical Interview for Diagnostic and Statistical Manual of Mental Disorders, Fourth Edition, Positive and Negative Symptom Scale and Schizophrenia Cognition Rating Scale. All participants were subjected to full medical and neurological examination and nociceptive reflex.

Results: There was no significant difference between cases and controls in nociceptive threshold, while it showed a significant difference at the subjective pain threshold as the case group needed a higher intensity of current to perceive as painful. There was a significant positive correlation between age, duration of $\mathrm{SCZ}$ and number of episodes and the nociceptive reflex. Also, there was a significant positive correlation between the negative symptoms of SCZ and cognitive impairment on one arm and the subjective pain threshold on the other arm.

Conclusion: The pain insensitivity in patients with SCZ is not related to physiological changes in the nociceptive pathway, but rather may be related to change in the experience and expression of pain. Older age, longer duration of disease, more frequent episodes, negative symptoms, and cognitive impairment, but not antipsychotic medications, all are positively correlated with the increase in subjective pain threshold.

Keywords: Nociceptive reflex; Pain threshold; Pain perception; Pain phenomenon; Schizophrenia

Manuscript submitted February 22, 2021, accepted April 14, 2021

Published online April 24, 2021

aDepartment of Neuropsychiatry, Faculty of Medicine, Menoufia University, Shebin El-Kom, Egypt

${ }^{b}$ Corresponding Author: Hosna Saad Soliman Elshony, Department of Neuropsychiatry, Faculty of Medicine, Menoufia University, Shebin El-Kom, Egypt. Email: hosna.saad28@gmail.com

doi: https://doi.org/10.14740/jnr656

\section{Introduction}

Schizophrenia (SCZ) is a severe chronic mental disorder which affects how a person thinks, feels and behaves. Patients with SCZ seem like they have lost touch with reality [1]. SCZ usually presents with long-standing delusions, non-affective psychosis and negative symptoms [2]. The structural and functional neuroanatomical deficits observed in $\mathrm{SCZ}$ using recent techniques have shed some light on the pathology of the disorder and also, highlighted the role of different brain areas and functional networks in the presence of different symptoms [3]. Sometimes, it is not clear what patients with $\mathrm{SCZ}$ can or cannot feel. It is acknowledged that patients with SCZ are very sensitive to stressful events. However, with this observation, there are also descriptions of reduced sensitivity to outside world. Those contradictory observations make it hard to know how patients react to different stimuli and situations, especially to unpleasant information [4].

Decreased pain sensitivity has been reported in patients with SCZ in the acute phase, as well as in stable phase. It has been observed that in patients with severe psychotic symptoms what is called psychotic analgesia occurs. The cause of hypoalgesia in patients with SCZ in stable phase is unknown [5]. Neuroleptic-induced analgesia, influence of neuropeptides with analgesic properties, lesions in the pain pathway, severe negative symptoms and cognitive impairment have been suggested. The hypothesis of an increased level of endogenous opioids was proposed but the results of the few studies are contradictory $[6,7]$.

So, we aimed in this study to investigate how patients with SCZ differ from normal persons in their perception and expression of pain and find explanations and factors contributing to these differences.

\section{Materials and Methods}

This case-control study was conducted on 36 participants, 24 patients with SCZ and 12 healthy individuals as a control group. They were selected from March 2017 to December 2018. The patients in the case group were selected carefully across the span of about 2 years from the patients who were seen for follow-up visits at the psychiatric outpatient clinic, or if hospitalized, they were scheduled for discharge based on the clinical assessment of symptoms.

The study was approved by the Ethical Committee in Jan- 
uary 2017. The study was performed in accordance with the Declaration of Helsinki.

\section{Patient selection}

\section{Inclusion criteria}

Both genders were included, and age ranged between 18 and 40 years. Patients were clinically fulfilling the criteria for the diagnosis of SCZ according to Diagnostic and Statistical Manual of Mental Disorders (DSM-5), in a full remission based on clinical assessment carried out by a senior psychiatrist, taking antipsychotic treatment in therapeutic doses. In this study, we selected patients on monotherapy only, antipsychotic drug doses were standardized using chlorpromazine equivalents, and such technique gave an estimation of therapeutic chlorpromazine dose equivalence for each antipsychotic drug and was used to statistically control the effects of medication.

\section{Exclusion criteria}

Exclusion criteria included age $<18$ years and $>40$ years, taking antidepressants, or anticonvulsive drugs in last 8 weeks, any mental disorders other than SCZ, presence of comorbid depressive symptoms on clinical examination, presence of any acute or chronic pain condition or taking any pain medication in the last $24 \mathrm{~h}$, peripheral neuropathy, limb trauma in the past, degenerative diseases of the musculoskeletal system or any neurological deficits, presence of intellectual disability or substance use disorder, presence of cardiac, respiratory, endocrine, or metabolic diseases, and a current diagnosis of diabetes or cancer.

\section{Methodology}

All cases and controls subjected to full medical history and full medical and neurological examination to detect any neurological or chronic medical condition that would lead to exclusion from the study.

Patients with SCZ were assessed with the following: 1) Diagnosis using the Structured Clinical Interview for Diagnostic and Statistical Manual of Mental Disorders, Fourth Edition (DSM-IV) (SCID-I) [8], Arabic version [9], which is a clinician-administered interview for use with psychiatric patients or with non-patient community subjects who are undergoing evaluation for psychopathology. The SCID-I was developed to provide broad coverage of psychiatric diagnoses according to DSM-IV. Arabic version used in this study was validated through its use in many studies that were conducted at the Institute of Psychiatry, Ain Shams University. 2) Assessment for positive and negative SCZ symptoms by Positive and Negative Symptom Scale (PANSS) [10], which comprises 30 items rated with a seven-point rating scale. It is a carefully defined and operationalized method that evaluates positive, negative and other symptom dimensions on the basis of a formal semi- structured clinical interview and other informational sources as caregivers and nursing staff. The positive scale (PANSS P) consists of seven items measuring symptoms that are super-added to a normal mental status, while the negative scale (PANSS N) consists of seven items measuring features absent from it. Finally, a third index, the General Psychopathology Scale (PANSS G), gauges the overall severity of SCZ disorder, including comorbid depressive and anxiety symptoms, by summation of the remaining 16 items. PANSS is a reliable tool to assess the severity of SCZ disorder. As 1 rather than 0 is given as the lowest score for each item, a patient cannot score lower than 30 for the total PANSS score. Scores are often given separately for the positive items, negative items, and general psychopathology. 3) Cognition assessment by Schizophrenia Cognition Rating Scale (SCoRS) [11] which is a 20-item interview-based assessment covering all cognitive domains, and takes approximately $12 \mathrm{~min}$ to complete. It is administered separately to the patient and to an informant (who is a person with regular contact with the patient in everyday situations), and in our study, we chose only members of the family like parents or spouses. The interviewer is asked to rate the patient's level of difficulty performing various cognitive functions on a four-point scale, with 4 being the most difficult and 1 being the least difficult. A rating of "not applicable" is also possible if a particular question does not apply to an individual patient. Upon completion of the 20 items, the interviewee is asked to give a global rating of the patient's cognitive functioning on a scale of 1 to 10 . After the interview is completed by both the patient and the informant, the interviewer ranks the patient on all the 20 items, and gives a global score based on the responses of both patient and informant as well as the interviewer's observations of the patient. The global rating reflects the overall impression of the patient's level of cognitive difficulty on a scale of $1-10$. Higher ratings indicate greater degrees of impairment.

All subjects underwent electrophysiological pain tests by nociceptive reflex [12]. In general aspects, all the experiments were performed by a senior neurologist (at the Department of Neurology, Menoufia University). Nerve conduction studies were performed using a TruTrace EMG device (Deymed Diagnostic, Czech Republic). The examiner was blinded to whether the participants were classified as cases or controls. During the testing session, the volunteers were lying in a bed, in a quiet room. A leg rest was placed under the knee to obtain a $30^{\circ}$ semiflexion. Each participant underwent a training session for the test in order to get familiar with the stimulation procedures before starting the data collection. All tests were applied to the right side of the body. Nociceptive reflex threshold was detected with surface electrodes placed near the lateral malleolus, at the anatomical surface area of the sural nerve. Using a computer-controlled current stimulator, a group of five square wave impulses was applied which was perceived as a single stimulus. Electromyographic reflex responses were recorded from the biceps femoris and the rectus femoris muscles. The current intensity was increased from $1 \mathrm{~mA}$ in steps of $0.5 \mathrm{~mA}$ until the nociceptive threshold was detected which was defined as the mean minimal stimulation intensity, at which a reflex motor response was elicited at $100 \%$. The subjective pain threshold was detected which was defined as the minimal current intensity 
Table 1. Socio-Demographic Data of the Case and Control Groups

\begin{tabular}{|c|c|c|c|c|}
\hline & Cases $(n=24)$ & Controls $(n=12)$ & Test of significance & P-value \\
\hline Age $($ mean $\pm S D)$, years & $27.54 \pm 5.509$ & $28.08 \pm 5.418$ & $\mathrm{t}=0.280$ & 0.781 \\
\hline Gender & & & $\chi^{2}=0.00$ & 1.000 \\
\hline Female, n (\%) & $10(41.7)$ & $5(41.7)$ & & \\
\hline Smoking & & & $\chi^{2}=0.223$ & 0.637 \\
\hline Education & & & $\chi^{2}=2.250$ & 0.522 \\
\hline Can read, n (\%) & $4(16.67)$ & $0(0.00)$ & & \\
\hline Primary, n (\%) & $5(20.83)$ & $3(25.0)$ & & \\
\hline High school, n (\%) & $10(41.66)$ & $6(50.0)$ & & \\
\hline
\end{tabular}

$X^{2}$ : Chi-square test. t: independent samples test. $P$ value $\leq 0.05$ is considered significant. $P$ value $<0.001$ is considered highly significant. SD: standard deviation.

perceived by participants as painful. The current stimulator applied these impulses at random time intervals, so the participant was unaware of the time the stimulus was applied.

\section{Statistical analysis}

Results were tabulated and statistically analyzed by using a personal computer using Microsoft Excel 2016 and SPSS v. 21 (SPSS Inc., Chicago, IL, USA). Statistical analysis was done using: descriptive: e.g. percentage (\%), mean and standard deviation; analytical: independent samples test, Mann-Whitney $\mathrm{U}$ test, one-way analysis of variance (ANOVA) test, KruskalWallis $\mathrm{H}$ test, Chi-squared $\left(\chi^{2}\right)$ test, McNemar test and Pearson correlation test. A P value less than 0.05 was considered statistically significant.

\section{Results}

Socio-demographic data for patients and controls are shown in Table 1 with no statistically significant difference between both groups.

Table 2 shows the clinical criteria for the SCZ group, age, onset and duration of illness, number of psychotic episodes along the course of the disorder, the score of PANSS for the patients which consists of a PANSS P with a mean of 18.96, a PANSS N with a mean of 18.75 and a PANSS G with a mean of 29.25, which indicates that all the patients in the case group were chosen to be in a stable mental state with no active positive or negative psychotic symptoms, while the interviewer global rating score of SCoRS with a mean of 2.63, indicating a non- to mild cognitive impairment.

On comparing electrophysiological findings in case and control groups, there was no significant difference at nociceptive threshold, while it showed a significant difference at the subjective pain threshold being higher in case group.

In case group, among the demographic factors, including age, gender, smoking and education, only age showed a significant positive correlation with the subjective pain threshold, and a highly significant positive correlation with the nociceptive threshold. Also, there was a highly significant positive correlation between the duration of SCZ disorder and number of psychotic episodes and both the subjective pain threshold

Table 2. Studied Patients' Clinical Criteria

\begin{tabular}{lll}
\hline & Mean \pm SD & Range (min. - max.) \\
\hline Age at onset of disorder, years & $22.58 \pm 4.169$ & $16-31$ \\
Duration of disorder, years & $4.96 \pm 3.043$ & $1-12$ \\
Number of episodes & $3.29 \pm 1.853$ & $1-7$ \\
PANSS P score & $18.96 \pm 3.316$ & $13-23$ \\
PANSS N score & $18.75 \pm 3.970$ & $12-27$ \\
PANSS G score & $29.25 \pm 5.318$ & $20-38$ \\
SCoRS global rating score & $2.63 \pm 0.576$ & $2-4$ \\
\hline
\end{tabular}

PANSS: Positive and Negative Syndrome Scale; PANSS P: Positive Scale; PANSS N: Negative Scale; PANSS G: General Psychopathology Scale; SCoRS: Schizophrenia Cognition Rating Scale; SD: standard deviation. 
Table 3. Correlation Between Demographic Data, PANSS and SCoRS and the Nociceptive Reflex

\begin{tabular}{lllll}
\hline & \multicolumn{2}{c}{ Subjective pain threshold } & & \multicolumn{2}{c}{ Nociceptive threshold } \\
\cline { 2 - 3 } Age & Correlation coefficient & P value & & Correlation coefficient \\
Gender & 0.377 & $0.023^{*}$ & 0.456 & $0.005^{* *}$ \\
Smoking & -0.310 & 0.066 & -0.177 & 0.301 \\
Education & 0.035 & 0.840 & 0.054 & 0.755 \\
Duration of disorder & -0.024 & 0.890 & 0.059 & 0.731 \\
Number of psychotic episodes & 0.594 & $0.002^{* *}$ & 0.589 & $0.002^{* *}$ \\
PANSS P & 0.545 & $0.006^{* *}$ & 0.553 & $0.005^{* *}$ \\
PANSS N & -0.178 & 0.406 & -0.291 & 0.168 \\
PANSS G & 0.413 & $0.045^{*}$ & 0.365 & 0.080 \\
SCoRS global rating & 0.403 & 0.051 & 0.225 & 0.291 \\
\hline
\end{tabular}

${ }^{*}$ Correlation is significant at the 0.05 level (two-tailed). ${ }^{* *}$ Correlation is highly significant at the 0.01 level (two-tailed). PANSS: Positive and Negative Syndrome Scale; PANSS P: Positive Scale; PANSS N: Negative Scale; PANSS G: General Psychopathology Scale; SCoRS: Schizophrenia Cognition Rating Scale.

and the nociceptive threshold. And there is a significant positive correlation between both the negative symptoms of SCZ and cognitive impairment and the subjective pain threshold (Table 3). Additionally, the current study shows that there is no effect of the different drugs on the subjective pain threshold or nociceptive threshold (Table 4).

\section{Discussion}

In people with SCZ, pain perception may be heightened or reduced as a result of delusions or other aspects of the disease. Moreover, it is often not possible to distinguish physical from mental pain, which makes pain control all the more important because, no matter its source, pain reduces quality of life in psychosis [13].

Many previous studies have been conducted to address the issue of pain perception and expression in patients with SCZ in the last two decades, with use of different modalities for assessment, on different numbers of patients in each study. In our study, we included 24 patients with SCZ and 12 healthy controls and we used the nociceptive reflex as the tool for pain assessment. We found that there was no significant difference between the case and control groups regarding the nociceptive threshold; however, there was significant difference regarding subjective pain threshold indicating that SCZ is associated

Table 4. Effect of Different Treatments on the Subjective Pain Threshold and Nociceptive Threshold

\begin{tabular}{|llll}
\hline Treatment & No. & Mean \pm SD & Test of significance \\
\hline Subjective pain threshold & & & P value \\
\hline Haloperidol & 4 & $40.00 \pm 20.06$ & \\
Risperidone & 7 & $48.00 \pm 19.86$ & \\
Olanzapine & 4 & $37.75 \pm 14.24$ & \\
Clozapine & 1 & 100.00 & \\
Aripiprazole & 3 & $39.33 \pm 9.23$ & \\
Amisulpride & 5 & $42.40 \pm 13.16$ & \\
Nociceptive threshold & & & \\
\hline Haloperidol & 4 & $30.0 \pm 2.82$ & \\
Risperidone & 7 & $38.29 \pm 11.85$ & \\
Olanzapine & 4 & $35.0 \pm 6.63$ & \\
Clozapine & 1 & 60.00 & \\
Aripiprazole & 3 & $34.00 \pm 5.29$ & \\
Amisulpride & 5 & $35.20 \pm 9.65$ & \\
\hline
\end{tabular}

F: analysis of variance test. ${ }^{*}$ Correlation is significant at the 0.05 level (two-tailed). ${ }^{* *}$ Correlation is highly significant at the 0.01 level (two-tailed). SD: standard deviation. 
with decreased pain sensitivity only when subjective pain assessment is tested as concluded in the systematic review done by Sakson-Obada, and that the physiological and basic affective responses to pain are intact, but the complex psychological elaboration seems to be impaired [6].

This is in agreement with Levesque et al, who used intermittent, transcutaneous stimulations of the left sural nerve, and found that, compared with controls, subjects with SCZ presented a specific experimental pain response profile, characterized by elevated sensitivity to acute pain but reduced sensitivity to prolonged pain. Group differences in subjective pain sensitization were not accompanied by group differences in nociceptive reflex activity, suggesting supraspinal origins to the change in pain experienced by subjects with SCZ [14]. Also Wojakiewicz et al found that patients with SCZ have a deficit of the identification and categorization of pain both in themselves and in others assessed by sensitivity to expressions of pain (STEP) test, pain video and situational pain questionnaire (SPQ) scores [15].

Jochum et al investigated the thresholds of warmth perception (WP), thermal pain onset (TPO) and thermal pain tolerance (TPT) in patients with acute SCZ and found that patients with SCZ showed significantly increased thresholds of WP and TPO relative to healthy controls. They believe that their findings can be attributed to information processing abnormalities of the disorder and that they are not specific to pain processing [16].

However, Zhou et al, combining psychophysics, electroencephalography (EEG) and functional magnetic resonance imaging (fMRI) techniques to investigate neural mechanisms of pain insensitivity in patients with SCZ, found that patients with SCZ were insensitive to nociceptive stimuli compared to healthy controls. Whereas perceived intensities to non-nociceptive somatosensory and auditory stimuli were not significantly different thus verifying the modality-specific nature of the modulation of nociceptive information processing in patients with SCZ and localizing the abnormality, using EEG and fMRI, to the thalamus and periaqueductal gray that are key nodes in ascending and descending pain pathways respectively, suggesting a possible contribution of cortical-subcortical dysfunction to the phenomenon [17].

This is in agreement with a previous study by De la FuenteSandoval et al, using fMRI, who examined the blood oxygen level-dependent (BOLD) changes induced by experimental pain-tolerance (endure) hot stimuli vs. non-painful stimuli during an acute psychotic episode in 12 drug-free patients with SCZ and in 13 gender- and age-matched healthy controls and confirmed that unmedicated patients with SCZ have a higher pain tolerance than controls, decreased activation in pain affective-cognitive processing regions (insula, posterior cingulate cortex and brainstem), and an over-activation of the primary sensory-discriminative pain processing region (S1) [18].

In comparison to other psychiatric disorders, Minichino et al compared pain processing in SCZ to bipolar I and II, and found that, compared to healthy subjects, patients with SCZ showed pain-processing and pain-perception abnormalities; however, patients with bipolar I showed only pain-processing abnormalities and patients with bipolar II did not differ for any of the variables investigated [19].
On the contrary, Girard et al found that patients with SCZ needed less pressure and a shorter duration of ischemia than controls to record moderate pain, and they felt more pain from a fixed pressure stimulus. Pain responses were unrelated to the clinical characteristics of the patients with SCZ, although hallucination production correlated with the pain felt during the fixed pressure test [20]. Also Urban-Kowalczyk et al found that there were no differences in nociceptive reflex testing among study groups but the mean $\beta$-endorphin concentration was about $20 \%$ higher in patients than in healthy controls [5].

In regards to the effect of demographic data on pain perception, we found there is a highly positive correlation between age and both the nociceptive threshold and the subjective painful threshold, which is consistent with other published results going back as far as the first half of the past century, when researchers found that the mean pain threshold of the subject group aged 10 - 22 years was significantly lower than the mean pain threshold of the group aged 23 - 44 years indicating that pain perception decreased with age $[7,21]$.

In our study, the results show a highly significant positive correlation between the duration of SCZ disorder and number of the psychotic episodes and both the subjective pain threshold and the nociceptive threshold. These results agreed with a previous study who found that there is a link between the occurrence of psychotic episodes and functional and structural abnormalities of the neural system mainly the anterior cingulate cortex and the anterior insular cortex which are the core regions of both the affective-motivational pain pathway and the salience network, such that a higher number of psychotic episodes correspond to greater network dysfunctions [19].

Also we found positive correlation between the negative symptoms of SCZ and the subjective pain threshold. They justify this fact that negative symptoms make the patient less likely to express their pain $[22,23]$. However, Urban-Kowalczyk et al found strong negative correlation between PANSS $\mathrm{N}$ scores and subjective pain threshold on the right lower limb as patients described even a very low current stimulus as painful [5]. This can be explained by the patient selection, as the author selected patients with severe negative symptoms and working memory disturbances. Also Levesque et al found that positive symptoms correlated negatively with pain threshold values among participants with SCZ, suggesting that distortions of thought and function relate to pain sensitivity in patients with SCZ [14].

On the other hand, we found no significant association between the general psychopathology scores of PANSS and subjective pain threshold, and nociceptive threshold. Although there are several studies connecting depression and anxiety symptoms to pain perception $[24,25]$. Our results can be explained by our selection of the patients, as there are only mild symptoms affecting the general condition of our case group. And so, our results are consistent with the results of a previous study [19].

Regarding cognition, we found positive correlation between the SCoRS and the subjective pain threshold, while there is no significant association between cognition and the nociceptive reflex which means that any impairment in the cognitive functions only affects the subjective perception of pain. 
These results agreed with a meta-analysis, explaining the decreased pain experience in patients with SCZ by several causes including impairments within the mediodorsal thalamus and hippocampus leading to affective and cognitive impairment which lead to decreased pain perception [26]. Also Minchino et al found that N1 and N2 amplitudes negatively correlated to social-cognition, and real-world functioning measures in the whole group of patients [19]. On the other hand, a previous study found a negative correlation between cognitive assessment and subjective pain threshold [5]. Also Wojakiewicz et al found that the patients' pain judgments were not correlated with their affective or cognitive empathic capacities [15] and Jochum et al found no correlation between pain perception and psychometric scales [16].

Our study shows no significant effect of the type of antipsychotic drugs on either the subjective pain threshold or the nociceptive threshold. Such results are consistent with several studies $[15,16,19]$. While another study talked about the antipsychotic medication normalizing pain perception in SCZ [18].

We acknowledge the following limitations in our study. First the small sample size is one of the biggest obstacles facing us and most of the studies about pain perception since many patients declined to consent to the procedures. Second, our method required the exclusion of any patient in acute psychotic episodes which limits our results as most prior literature noticed the altered pain perception during acute episodes.

\section{Conclusion}

The pain insensitivity in patients with SCZ is not related to physiological changes in the nociceptive pathway, but rather may be related to pain affective-cognitive processing abnormalities. Older age, longer duration of disease, more frequent episode, negative symptoms, and cognitive impairment, but not antipsychotic medications, all are positively correlated with increase subjective pain threshold. More studies need to address phenomena of pain in SCZ, including pain perception during acute episodes, the effect of treatment itself on pain phenomena and the effect of duration of disorder on pain perception.

\section{Acknowledgments}

The authors would like to thank Dr Marwa Khalil for sharing in data collection.

\section{Financial Disclosure}

None to declare.

\section{Conflict of Interest}

None to declare.

\section{Informed Consent}

Written informed consent was obtained from the patient.

\section{Author Contributions}

HE contributed to literature search, data acquisition and analysis, manuscript preparation and editing. LH contributed to study design, manuscript preparation and data analysis. All authors have read and approved the manuscript.

\section{Data Availability}

The data supporting the findings of this study are available from the corresponding author upon reasonable request.

\section{Abbreviations}

PANSS: Positive and Negative Symptom Scale; PANSS P: Positive Scale; PANSS N: Negative Scale; PANSS G: General Psychopathology Scale; DSM: Diagnostic and Statistical Manual of Mental Disorders; SCoRS: Schizophrenia Cognition Rating Scale; SCZ: schizophrenia

\section{References}

1. The National Institute of Mental Health (NIMH). Schizophrenia. 2016. Retrieved from: www.nimh.nih.gov.

2. Alkhadhari S, Al Zain N, Darwish T, Khan S, Okasha T, Ramy H, Tadros TM. Use of second-generation antipsychotics in the acute inpatient management of schizophrenia in the Middle East. Neuropsychiatr Dis Treat. 2015;11:915-924.

3. Wylie KP, Tregellas JR. The role of the insula in schizophrenia. Schizophr Res. 2010;123(2-3):93-104.

4. Duval CZ, Goumon Y, Kemmel V, Kornmeier J, Dufour A, Andlauer O, Vidailhet P, et al. Neurophysiological responses to unpleasant stimuli (acute electrical stimulations and emotional pictures) are increased in patients with schizophrenia. Sci Rep. 2016;6:22542.

5. Urban-Kowalczyk M, Pigonska J, Smigielski J. Pain perception in schizophrenia: influence of neuropeptides, cognitive disorders, and negative symptoms. Neuropsychiatr Dis Treat. 2015;11:2023-2031.

6. Sakson-Obada O. Pain perception in people diagnosed with schizophrenia: where we are and where we are going. Psychosis. 2017;9(4):358-366.

7. Chapman WP, Jones CM. Variations in cutaneous and visceral pain sensitivity in normal subjects. J Clin Invest. 1944;23(1):81-91.

8. First MB, Spitzer RL, Gibbon MW, et al. Structured clinical interview for DSM-IV - clinical version (SCID-CV) [user's guide and interview].

9. El Missiry A, Sorour A, Sadek A, et al. Homicide and 
psychiatric illness, an Egyptian study (MD thesis). Cairo: Faculty of Medicine, Ain Shams University. 2003.

10. Kay SR, Fiszbein A, Opler LA. The positive and negative syndrome scale (PANSS) for schizophrenia. Schizophr Bull. 1987;13(2):261-276.

11. Keefe RS, Davis VG, Spagnola NB, Hilt D, Dgetluck N, Ruse S, Patterson TD, et al. Reliability, validity and treatment sensitivity of the Schizophrenia Cognition Rating Scale. Eur Neuropsychopharmacol. 2015;25(2):176-184.

12. Guieu R, Samuelian JC, Coulouvrat H. Objective evaluation of pain perception in patients with schizophrenia. Br J Psychiatry. 1994;164(2):253-255.

13. Stubbs B, Gardner-Sood P, Smith S, Ismail K, Greenwood K, Patel A, Farmer R, et al. Pain is independently associated with reduced health related quality of life in people with psychosis. Psychiatry Res. 2015;230(2):585591.

14. Levesque M, Potvin S, Marchand S, Stip E, Grignon S, Pierre L, Lipp O, et al. Pain perception in schizophrenia: evidence of a specific pain response profile. Pain Med. 2012;13(12):1571-1579.

15. Wojakiewicz A, Januel D, Braha S, Prkachin K, Danziger $\mathrm{N}$, Bouhassira D. Alteration of pain recognition in schizophrenia. Eur J Pain. 2013;17(9):1385-1392.

16. Jochum T, Letzsch A, Greiner W, Wagner G, Sauer H, Bar KJ. Influence of antipsychotic medication on pain perception in schizophrenia. Psychiatry Res. 2006;142(23):151-156.

17. Zhou L, Bi Y, Liang M, Kong Y, Tu Y, Zhang X, Song Y, et al. A modality-specific dysfunction of pain processing in schizophrenia. Hum Brain Mapp. 2020;41(7):17381753.

18. de la Fuente-Sandoval C, Favila R, Gomez-Martin D, Pellicer F, Graff-Guerrero A. Functional magnetic resonance imaging response to experimental pain in drug-free patients with schizophrenia. Psychiatry Res. 2010;183(2):99104.

19. Minichino A, Delle Chiaie R, Cruccu G, Piroso S, Di Stefano G, Francesconi M, Bersani FS, et al. Pain-processing abnormalities in bipolar I disorder, bipolar II disorder, and schizophrenia: A novel trait marker for psychosis proneness and functional outcome? Bipolar Disord. 2016;18(7):591-601.

20. Girard M, Plansont B, Bonnabau H, Malauzat D. Experimental pain hypersensitivity in schizophrenic patients. Clin J Pain. 2011;27(9):790-795.

21. Haslam DR. Age and the perception of pain. Psychon Sci. 1969;15(2):86-87.

22. Bonnot O, Anderson GM, Cohen D, Willer JC, Tordjman S. Are patients with schizophrenia insensitive to pain? A reconsideration of the question. Clin J Pain. 2009;25(3):244-252.

23. Engels G, Scherder E. Clinical pain in schizophrenia: a forgotten area. In: Pickering G., Gibson S, editors. Pain, Emotion and Cognition. A Complex Nexus 1st ed. Springer, Cham, Switzerland: Springer International Publishing. 2015. p. 221-230.

24. Tikasz A, Tourjman V, Chalaye P, Marchand S, Potvin S. Increased spinal pain sensitization in major depressive disorder: A pilot study. Psychiatry Res. 2016;246:756761.

25. Vaughan S, Failla MD, Poole HM, et al. Pain processing in psychiatric conditions: a systematic review. Rev Gen Psychol. 2019;23(3):336-358.

26. Stubbs B, Gaughran F, Mitchell AJ, De Hert M, Farmer R, Soundy A, Rosenbaum S, et al. Schizophrenia and the risk of fractures: a systematic review and comparative metaanalysis. Gen Hosp Psychiatry. 2015;37(2):126-133. 